\title{
Small Bowel Perforations: What the Radiologist Needs to Know
}

\author{
Giuseppe Lo Re, MD, Francesca La Mantia, MD, Dario Picone, MD, \\ Sergio Salerno, MD, Federica Vernuccio, MD, and Massimo Midiri, MD
}

\begin{abstract}
The incidence of small bowel perforation is low but can develop from a variety of causes including Crohn disease, ischemic or bacterial enteritis, diverticulitis, bowel obstruction, volvulus, intussusception, trauma, and ingested foreign bodies. In contrast to gastroduodenal perforation, the amount of extraluminal air in small bowel perforation is small or absent in most cases. This article will illustrate the main aspects of small bowel perforation, focusing on anatomical reasons of radiological findings and in the evaluation of the site of perforation using plain film, ultrasound, and multidetector computed tomography equipments. In particular, the authors highlight the anatomic key notes and the different direct and indirect imaging signs of small bowel perforation.

Semin Ultrasound CT MRI I:III-n (C) 2015 Elsevier Inc. All rights reserved.
\end{abstract}

\section{Introduction}

S mall bowel perforation is an acute emergency condition $\checkmark$ due to a transmural lesion, which affects the full thickness of the bowel wall with the communication of the intestinal lumen with the abdominal cavity and the leakage of intestinal content. ${ }^{1,2}$ Early diagnosis, as well as prompt surgical treatment, are essential to reduce the morbidity and the mortality. ${ }^{3,4}$ However, perforation of the small bowel is not a common cause of acute abdomen and can have a traumatic or nontraumatic etiology. ${ }^{2-8}$ The traumatic perforations of small bowel are more frequent than nontraumatic ones. ${ }^{5-7,9-13}$ In particular, small bowel traumatic perforation is the third in frequency among abdominal traumatic perforations following liver and spleen ones; these lesions are rarely isolated. ${ }^{9-13}$

The origin of nontraumatic perforations may be infectious (typhoid fever, HIV, tuberculosis, hook worms; most common in developing countries) or noninfectious (ischemic conditions, Meckel diverticulitis, Crohn's disease, tumors, iatrogenic causes, foreign bodies, and mechanical conditions). ${ }^{8-12}$ Clinical findings of small bowel perforation are usually not specific, sudden acute abdominal pain, vomiting, anorexia, and nausea. In this article, we report our experience, we retrospectively

Section of Radiological Sciences, DIBIMED, University of Palermo, Palermo, Italy.

Address reprint requests to Giuseppe Lo Re, MD, Section of Radiological Sciences, DIBIMED, University of Palermo, Via Cristofaro Scobar 1, Palermo 90145, Italy. E-mail: giuseppe.lore12@gmail.com evaluated all CT reports from 2011-2015 searching for "Intestinal Perforation" and reviewed all the images of exams in which small bowel perforation was diagnosed.

\section{Anatomic Key Notes}

The small bowel lies between the stomach and the large bowel and includes the duodenum, jejunum, and ileum. Embryologically, it develops mainly from the midgut; however, the proximal part of the duodenum develops from the caudal foregut. ${ }^{14}$ The junction of embryological foregut and midgut is marked by the site of the major duodenal papilla on the medial wall of the second part of the duodenum. ${ }^{14}$

The peritoneum is a thin serous membrane consisting of 2 layers that line the abdominal wall (parietal peritoneum) and cover some of the abdominal organs (visceral peritoneum). ${ }^{14}$ The peritoneal cavity is a virtual space between the parietal peritoneum and the visceral one. ${ }^{14}$ On the other hand, abdominal organs located behind the intraperitoneal space are called "retroperitoneal." The duodenum is intraperitoneal for the first $2-2.5 \mathrm{~cm}$, whereas the remaining duodenum is retroperitoneal; jejunum and ileum are intraperitoneal organs.

Peritoneal ligaments, known as mesentery and omentum divide the peritoneum into 2 compartments, the greater and the lesser sac (Fig. 1).

The peritoneal cavity is divided by the transverse mesocolon in 2 great spaces: the supramesocolic and the inframesocolic spaces. The first is the one above the root of the transverse 


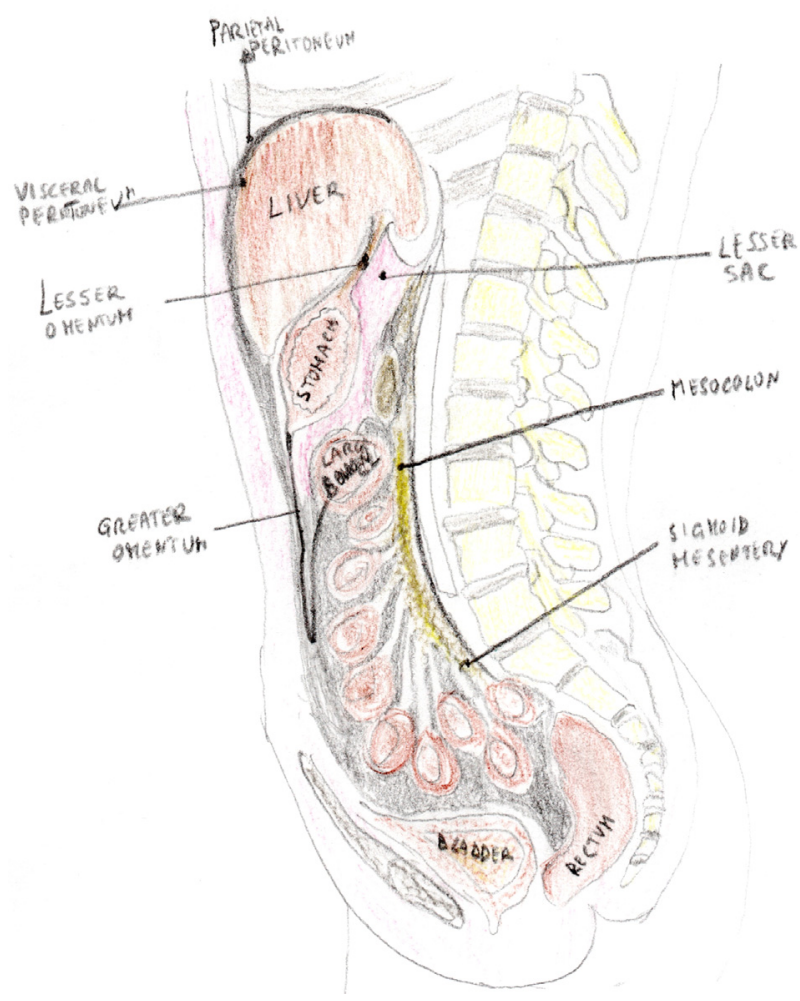

Figure 1 Sagittal view of the abdomen showing main abdominal key notes. (Color version of figure is available online.)

mesocolon and contains liver, stomach and spleen, whereas the latter is located below the root of the transverse mesocolon and contains the small bowel loops.

The supramesocolic space may also be divided into right and left supramesocolic spaces. ${ }^{14}$ The first includes the right subphrenic space, anterior right subhepatic space, posterior right subhepatic space (also known as Morrison pouch), and the lesser sac. ${ }^{14}$ The left supramesocolic space includes anterior and posterior left perihepatic space, anterior and posterior (or perisplenic) left subphrenic space. ${ }^{14}$

According to these anatomic key notes, it is important to keep in mind these useful tips and tricks ${ }^{14,15}$ :

- Gastric or duodenal perforation will result in air bubbles in the supramesocolic compartment. Air bubbles may travel first in the gastroduodenal ligament, then from the hepatoduodenal ligament into the fissure for ligamentum venosum and be detected adjacent to the portal vein.

The organs implied in supramesocolic perforation can be stomach and duodenum and the etiology are usually peptic ulcer disease, neoplasia, and postoperative anastomotic leaks. In particular, penetrating ulcers involving the anterior wall of the stomach or duodenum may perforate directly into the peritoneal cavity, although the ones posterior wall gastric ulcers perforate into the lesser sac.

The inframesocolic space is divided in 4 compartments: right paracolic gutter, right inframesocolic space (between mesentery and ascending colon), left inframesocolic space (between mesentery and discending colon), and left paracolic gutter. ${ }^{14}$ The pelvis is the most lower space of the peritoneal cavity and contains bladder, rectum, sigma, and riproductive system. The pelvic space is composed of paravescical space, in men there is the rectovescical space, in woman there are uterovescical space and uterorectal space (Douglas's space) ${ }^{14}$ :

- Air bubbles in the inframesocolic compartment may indicate a perforation of small or large bowel. ${ }^{14}$ However, the incidence of small bowel perforations is low and may be of various origin (ischemic, inflammatory, infectious, postoperative, bowel obstruction, volvulus, and intussusceptions) ${ }^{8-12}$ and

- perforation of the second and third duodenum segments may result in gas bubbles located just in the retroperitoneal space. The most common location of retroperitoneal gas is the anterior pararenal space and, in this case, gas typically outlines the lateral border of the psoas muscle.

Retroperitoneal cavity contains suprarenal glands, aorta or inferior vena cava, duodenum (second and third part), pancreas, ureteres, colon (ascending and descending), kidneys, esophagus, and rectum. ${ }^{14}$

\section{Imaging Findings}

Patients with acute abdominal pain and clinical suspicion of bowel perforation, are usually firstly submitted to x-ray. The key direct radiographic sign of bowel perforation is free abdominal gas. ${ }^{1}$ However, free air is differently distributed on plain films in the abdominal cavity according to patient positioning and to the perforation site. ${ }^{1,16-18}$

The sensitivity of plain abdominal radiography ranges from $50 \%-70 \%$ for gastrointestinal perforation, though these rates are lower in cases of early perforations with less than $1 \mathrm{ml}$ of gas. ${ }^{1,2}$

Abdominal ultrasonography (US) is often performed in patients with abdominal pain, but it is less sensitive than computed tomography (CT) to reveal the cause and the site of the intestinal perforation. ${ }^{1,16,19}$

Direct ultrasonographic signs of small bowel perforation are: strong reverberation anteriorly to the liver surface, shifting phenomenon and "scissors maneuver," enhancement of peritoneal stripe. Indirect ultrasonographic signs of bowel perforation are: presence of intraperitoneal free fluid, decreased bowel motility. ${ }^{18-20}$

$\mathrm{CT}$ is more sensitive compared to plain abdominal films to reveal small amounts of free air and multidetector row CT allows in many cases the identification of intestinal perforation site, may provide many suggestive diagnostic and prognostic features of the causing disease and other findings that may occur in emergency as hemoperitoneum. ${ }^{21-27}$

Direct CT signs of intestinal perforations are: free gas in the abdominal cavity, visible transmural lesion of intestinal wall, extraluminal leakage of orally administered contrast 


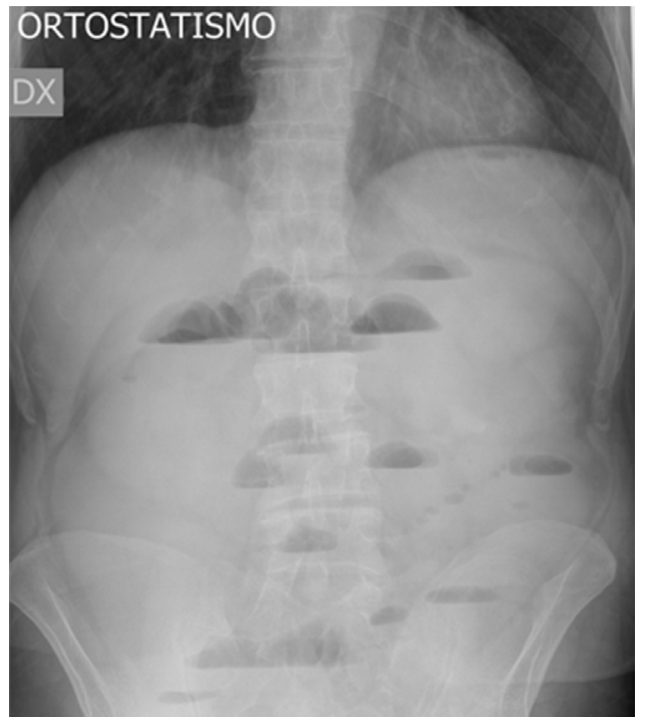

Figure 2 Abdominal upright radiograph performed in a 60-year-old male shows multiple air-fluid levels in the small bowel. The radiologist raised the suspicious of air under the left diaphragmatic dome (oval shape); CT was performed and confirmed the presence of small bowel occlusion and perforation.

medium. ${ }^{16,17,27}$ In our department, oral contrast material is not used for this indication.

Indirect CT signs of intestinal perforations are misty mesentery, free fluid in the abdominal cavity, bowel wall thickening, and extraluminal fecal matter ("dirty mass"). ${ }^{17}$

\section{Plain Film Technique}

The complete x-rays series includes upright abdominal plain film, supine anteroposterior abdominal plain film, and upright posteroanterior chest plain film. ${ }^{1,16,17}$

As reported in the introduction, the key direct radiographic sign of bowel perforation is free abdominal gas (intraperitoneal and retroperitoneal). In the upright abdominal and chest films free air is trapped under right or left hemidiaphragm, or both right and left hemidiaphragm (Figs. 2 and 3).

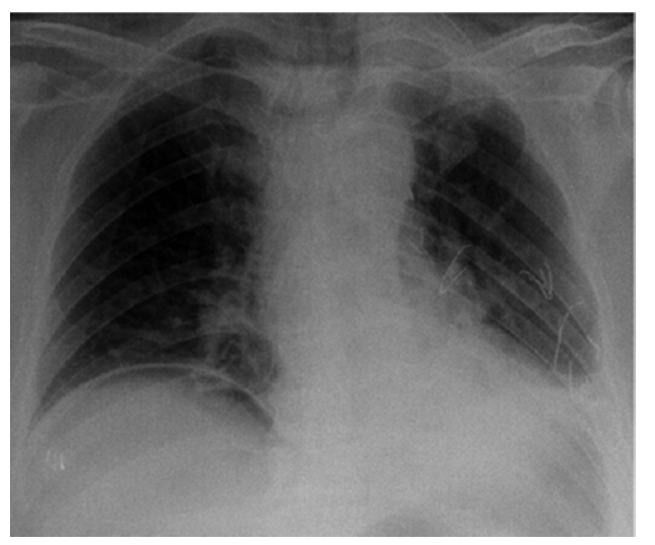

Figure 3 Chest x-ray performed as postoperative routine shows the presence of air under the right diaphragmatic dome.
Supine abdominal plain films can reveal ${ }^{16,28}$ :

- Rigler's sign, also known as double wall sign, characterized by the presence of air on both the luminal and peritoneal side of the bowel wall;

- cap of doge sign, when there is air in Morrison's pouch, which presents as a triangular gas density;

- lucent liver sign, characterized by a reduction of hepatic radiodensity;

- free air in subhepatic and anterior space;

- cupola sign, which forms because of the presence of free air under the central tendon of the diaphragm in the midline;

- falciform ligament sign, which is characterized by the visualization of falciform ligament by free gas on either side of the ligament;

- free air in lesser sac;

- football sign, a large round black area in the abdomen due to a large volume of free gas risen to the front of the peritoneal cavity;

- ligamentum teres sign, which is characterized by air outlining fissure of ligamentum teres hepatis, which is the posterior free edge of falciform;

- "inverted V" sign, due to the presence of free air outlining the lateral umbilical ligaments forming an "inverted V" in the lower abdomen;

- urachus sign: air contrasted urachus appears as a radiopacity line between bladder and umbilicus; and

- pneumomesocolon, pneumomentum.

Other possible signs are visible gallbladder sign, visible lower cardiac border sign, bubbles to the upper hepatic surface, diaphragmatic muscle slip sign, visible transverse mesocolon, and visible small bowel mesentery.

Other 2 projections can be performed, supine lateral abdominal plain (free gas is trapped between anterior abdominal wall and bowel loops) and lateral chest plain (free air trapped between the liver and the abdominal wall). ${ }^{16,28}$

However, compared to these latter projections, upright abdominal plain film and lateral chest plain film are more sensitive to reveal small amounts of air.

Other radiographic features of perforation can be pneumomediastinum and subcutaneous emphysema. However, free abdominal air may not always visible on abdominal plain films, mainly in case of small amounts of air (1 ml), air contained by adjacent organs, bowel loop without air and leakage of only fluid, re-absorbed gas ${ }^{19}$; moreover, its distribution is not specific (in small bowel perforations air can be distributed in supra and submesocolic space), and the site of perforation is not detected.

However, there are the following conditions that may mimic the presence of free air in the abdominal cavity (also known as pseudopneumoperitoneum):

- Pneumomediastinum may mimic cupola sign;

- portal pneumatosis or intestinal pneumatosis may mimic free abdominal air ${ }^{29,30}$; 


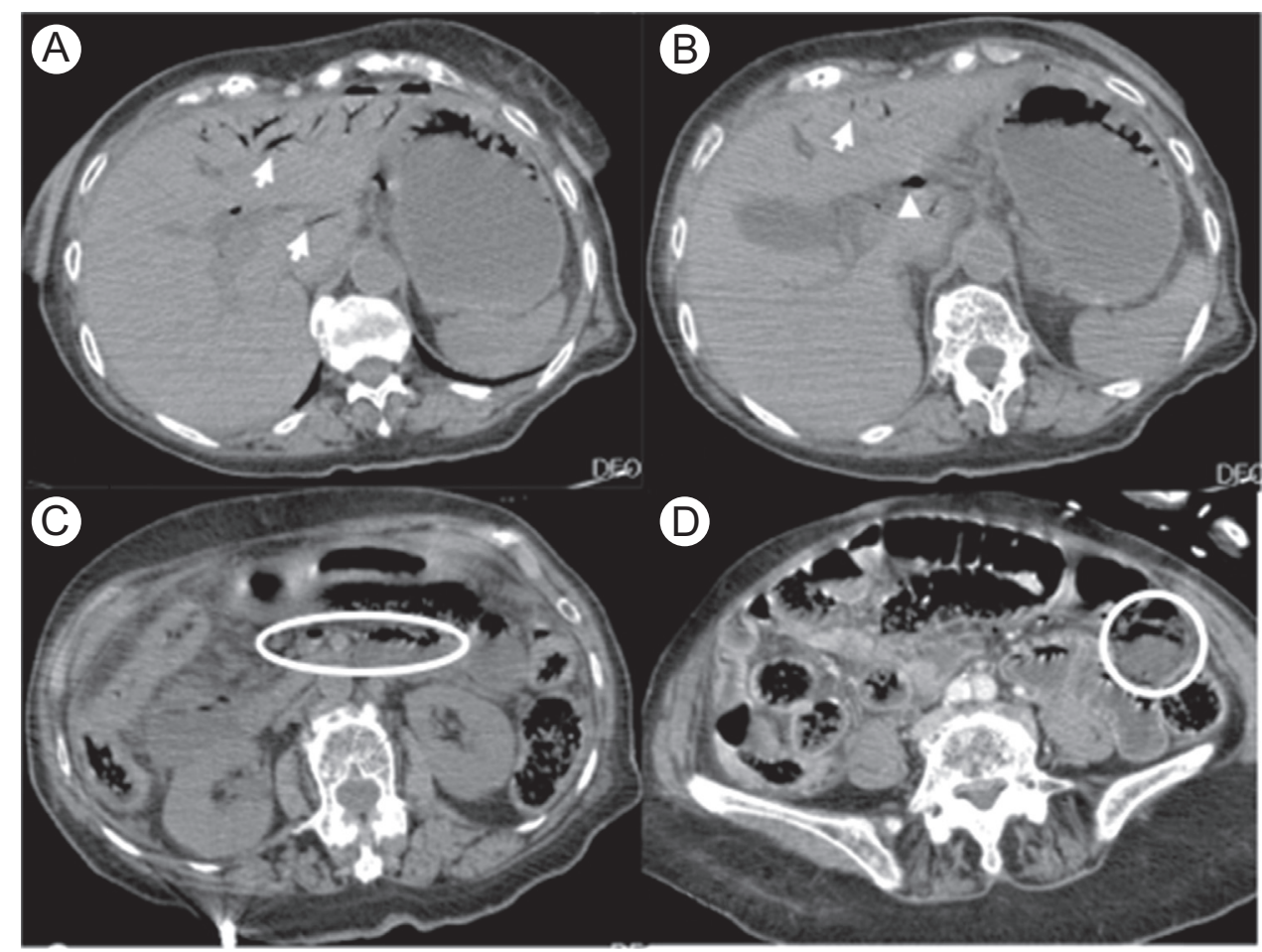

Figure 4 An 85-year-old female referred to the Emergency Department for abdominal pain and later to the Department of Radiology for clinical suspicion of ischemia. (A and B) Evidence of a gas bubble next to the portal vein (arrowhead) and portal pneumatosis (arrows) of the left branches and in the caudate lobe. The same patients showed air in the mesenteric and ileocolic veins (C, oval) and intestinal pneumatosis of a small bowel (D, circle), associated with distension and air-fluid air of the small bowel.

- Chilaiditi's phenomenon ${ }^{31}$ : gas forms a crescent shape under the right hemidiaphragm, which is thickened; this phenomenon occurs in patients with small liver or flattened diaphragms, in whom these conditions are responsible of the creation of a space within the upper abdomen above the liver and this space may be filled by bowel, whose air content may mimic free gas; and

- false Rigler's sign due to the presence of adjacent bowel loops that contain air; the imaging appearance is the presence of air on both sides of the bowel wall, as in Rigler sign. ${ }^{28}$

\section{US Technique}

US is often performed in patients with abdominal pain and to avoid CT radiation in children or pregnant women. Recently, some studies proved the role of contrast-enhanced US in the lesion detection and grading in patients with minor abdominal trauma. $^{32,33}$

Direct US signs of small bowel perforation are strong reverberation anteriorly to the liver surface, shifting phenomenon and "scissors maneuver" (when the patient changes position the air moves to the highest portion of abdominal cavity). ${ }^{34}$ The scissors maneuver is the following: applying a

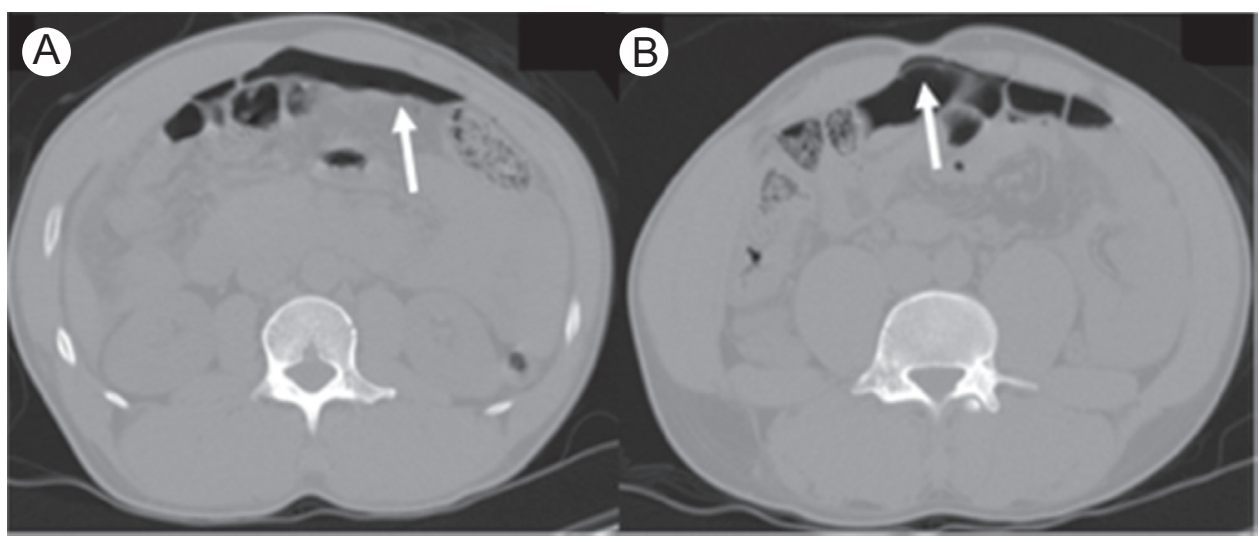

Figure 5 Abdominal CT shows the presence of free abdominal air (A and B, arrow) in the abdominal cavity due to large bowel perforation. 


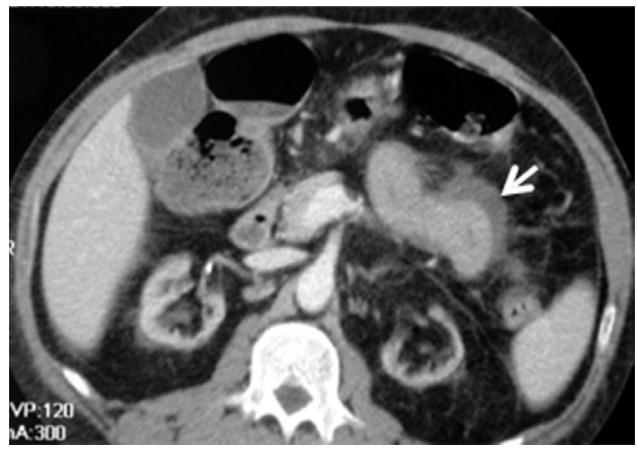

Figure 6 Contrast-enhanced abdominal CT image of a patient with a jejunal loop perforation. Evidence of peritoneal fluid next to the jejunal loop (arrow).

pressure on the abdomen with the probe there is an air shifting and consequently an attenuation of the reverberation, whereas the reverberation increases when the pressure is stopped. ${ }^{34}$

Indirect US signs are presence of intraperitoneal free fluid and decreased bowel motility. Patients are studied in the supine and semilateral positions; patient's cooperation and his habitus represent important limits of US. ${ }^{19,25}$ This technique is certainly less sensitive than CT to detect the cause and the site of the intestinal perforation.

\section{CT Technique}

CT is more sensitive compared to plain abdominal film to reveal small amounts of free air and multidetector row CT allows in many cases the identification of intestinal perforation site $^{35}$ (Figs. 4 and 5).

Direct CT signs of intestinal perforations are free gas, extraluminal leakage of orally administered contrast medium, and visible transmural lesion of intestinal wall. 2,17,27

Considering the first direct sign, in small bowel perforations free air can be trapped in supra and submesocolic space. Although CT is more sensitive than plain film to reveal small amount of air, in some cases free air is not present as in cases of small bowel perforations. ${ }^{1,5,6,17}$ In fact, in small bowel perforation free air is absent in $50 \%$ of cases. ${ }^{2}$
Considering the second direct sign, that is the extraluminal leakage of orally administered contrast medium, in our department, the oral administration of contrast material is not used. Some authors discuss about the disadvantages of the oral administration of contrast material, possible adverse reactions, longer CT examination time, impossibility to reveal perforations of the anterior intestinal wall because of decreased bowel motility in paralytic ileus, and the higher amount of radiations; moreover, imaging reliability is decreased by the absence of bowel cleansing in emergency and by the fact that the presence of oral contrast medium may obscure bowel wall enhancement. ${ }^{36,37}$

Another direct sign of bowel perforation is the discontinuity of bowel wall. ${ }^{2}$ This sign is not easily detectable; however, the presence of air bubbles, small amounts of free fluid or segmental bowel thickening near the site of a transmural lesion can be helpful. 2,6

Indirect signs of bowel perforation are misty mesentery, free fluid (Fig. 6), wall tickening (Fig. 7), and extraluminal fecal matter ("dirty mass"). ${ }^{17,27,38}$ The "dirty mass" sign is the most frequent one in rectum perforation. ${ }^{38}$

Misty mesentery is a common but nonspecific sign of bowel perforation; it is defined as an increased attenuation of the mesentery on CT due to the infiltration of the mesenteric fat with inflammatory cells, fluid (edema, lymph, and blood), malignant cells, and fibrosis. ${ }^{39}$

Another indirect sign of bowel perforation is intraperitoneal free fluid (Fig. 8); it can be the indirect sign of transmural lesion of the bowel wall but it also a not specific sign of peritoneum's reaction. ${ }^{24}$ In small bowel perforations free air is often absent, while free fluid, associated with the clinical suspect of bowel perforation, represent an important indirect sign of bowel injury. ${ }^{24}$

Other less frequent CT signs of perforation are:

- Intestinal pneumatosis: characterized by the presence of air bubbles in the intestinal wall; this condition may be because of advanced intestinal ischemia ${ }^{28,29}$;

- portomesenteric pneumatosis: characterized by the presence of air bubbles in the mesenteric vein, portal vein and in its branches; it is mainly due to intestinal ischemia and is indicative of poor prognosis; and

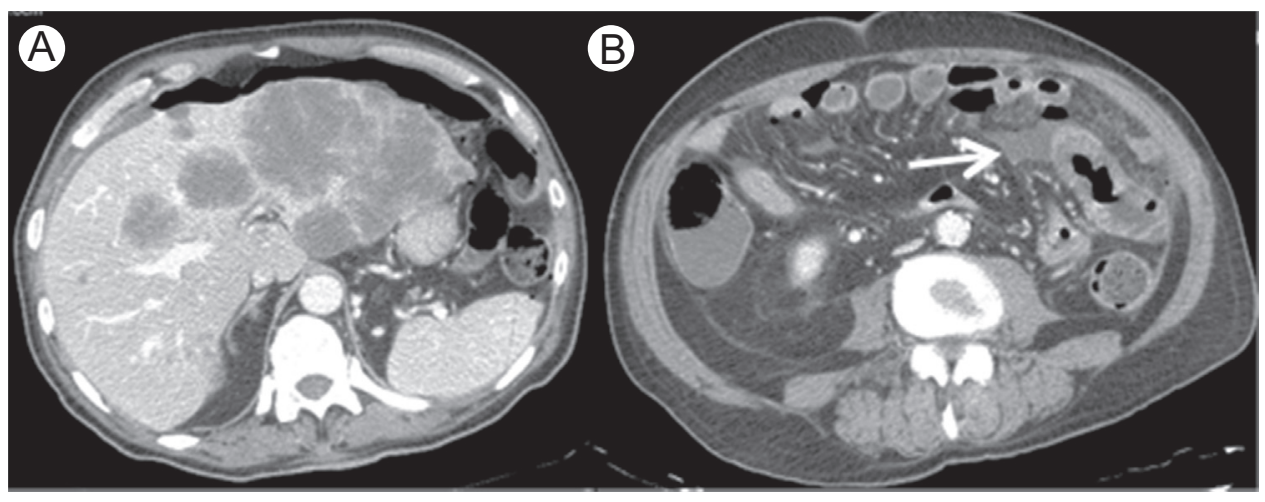

Figure 7 Contrast-enhanced abdominal CT image of a patient with hepatic metastases (A). Evidence of peritoneal fluid and free air anterior to the liver surface (A). Irregular thickening and narrowing of a small bowel loop with free fluid (arrow) and air bubble into the adjacent mesenteric fold (B). 


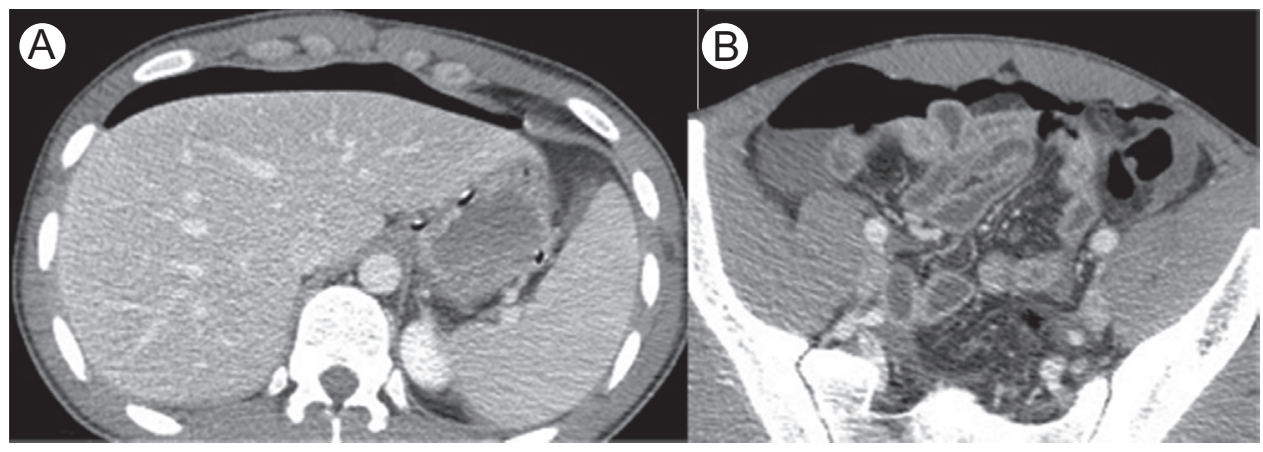

Figure 8 Contrast-enhanced abdominal CT images of a patient with a posttraumatic jejunal loop perforation. Presence of pneumoperitoneum and peritoneal fluid (A and B).

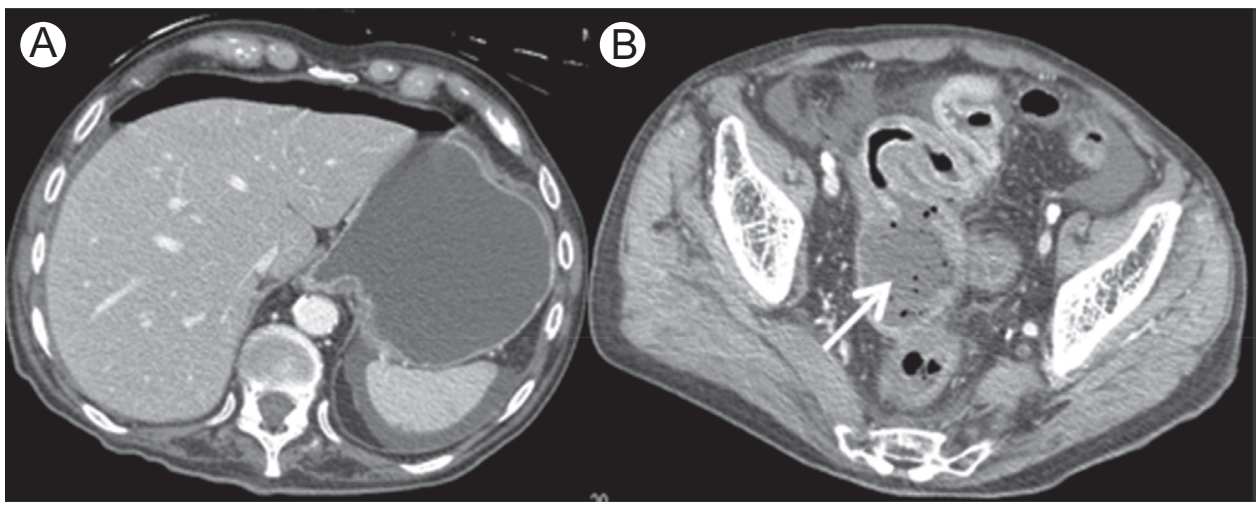

Figure 9 Contrast-enhanced abdominal CT of a patient with ileal loop perforation. Presence of peritoneal fluid and pneumoperitoneum anterior to the liver surface (A). In the pelvis, evidence of bowel wall discontinuity with the development of an abscess, demonstrated by a low-density collection with air bubbles (B, arrow).

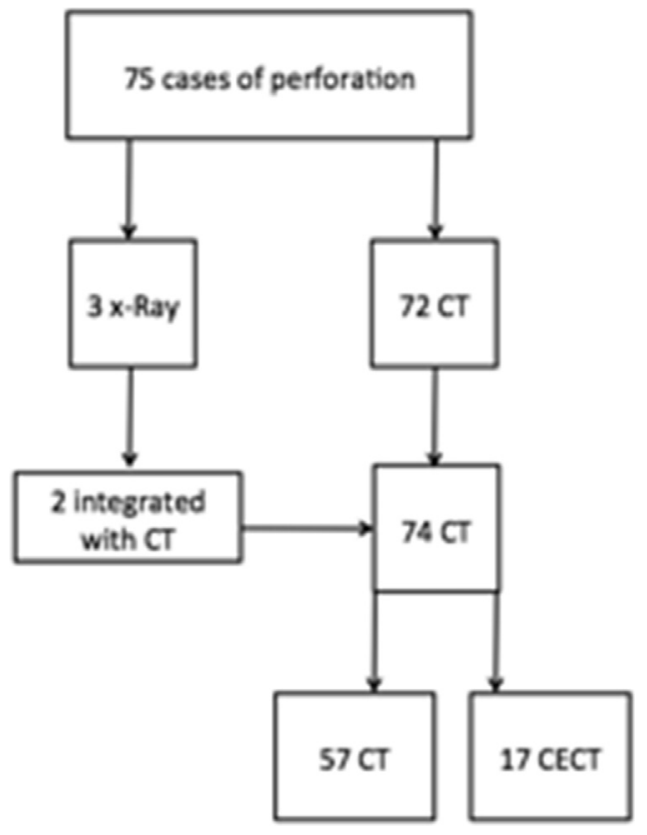

Figure 10 Flow chart showing our experience in the imaging diagnosis of intestinal perforation.
- abdominal ascesses, that may be encountered not only in gastrointestinal perforation but also in postoperative bacteremia and trauma (Fig. 9).

A multislices CT (MSCT) scanner should be chosen, if available, because of its high spatial reconstruction and the consequent possibility to obtain multiplanar and minIP images that turn out to be quite useful for the radiologist to find the site of perforation and evaluate its entity. In particular, Slab minIP images have a higher sensibility in the depiction of air bubbles, especially in case of minimal amount of free air in abdomen.

Yet, radiologists need to have good experience in the use of workstation and protocols of images reconstruction.

\section{Our Experience}

\section{Materials and Methods}

We retrospectively evaluated all plain films and CT imaging reports of our institution from 2011-2015 searching for "Intestinal Perforation" in the picture archiving and communication system. The inclusion criteria were patients with diagnosis of small bowel perforation who had undergone 
Table Computed Tomography Signs of Perforation Encountered in Our Experience

\begin{tabular}{lll}
\hline CT Sign & No. of Patients & Percentage (\%) \\
\hline Free gas & 26 & 100 \\
Transmural lesion & 7 & 26.9 \\
Misty mesentery & 19 & 73.1 \\
Free fluid & 14 & 53.8 \\
Wall thickening & 18 & 69.2 \\
\hline
\end{tabular}

radiological investigations before surgery. The exclusion criteria were the absence of surgery and radiological investigations before surgery.

Images were always available. All CT images were retrospectively analyzed on a dedicated professional workstation (PACS-Impax, Agfa-Gevaert, Milan, Italy), by 2 experienced abdominal radiologists. Images of the selected examinations were reviewed and the following $\mathrm{x}$-rays and CT signs were evaluated: direct signs (free gas, break of intestinal wall) and indirect signs (misty mesentery, free fluid, wall thickening).

The sites of the CT findings were compared with the site of perforation observed at surgery.

\section{Technical Parameters}

The studies were done through an MSCT (GE BRIGHTSPEED 16, Milwahukee). CT scanning was performed from the diaphragm to the pubic symphysis using the following acquisition parameters: slice thickness $=1.25$ $2.5 \mathrm{~mm}$, reconstruction interval $=1.25 \mathrm{~mm}, 120 \mathrm{kV}$, $250-300 \mathrm{mAs}$, pitch $=1.5$. The intravenous contrast agent administered, when used, was habitually a nonionic uroangiographic contrast material with concentration of $320-350 \mathrm{mgl} / \mathrm{ml}$.

\section{Results}

In our experience from 2011-2015, the diagnosis of intestinal perforation appeared in 75 cases (42 males and 33 females; aged $72.7 \pm 17.4$ ) (Fig. 10). In 3 cases, plain film had been performed and 2 cases were integrated with MSCT. From the review of the x-rays images, we found free gas under right and left dome of diaphragm in 2 cases, in 1 case free air was collected under right dome of hemidiaphragm.

In 74 cases, CT was performed and in 17 cases intravenous iodinated contrast material was administered.

In all patients, CT allowed the identification of the site of perforation, (stomach or duodenum: 7 cases; small bowel: 26 cases; and large bowel: 41 cases).

CT signs encountered were free gas in 26 cases, visible transmural lesion in 7 cases, misty mesentery in 19 cases, free fluid in 14 cases, wall thickening in 18 cases (Table).

Gas bubbles adjacent to the perforated wall were observed in all patients.

The diagnosis of small bowel perforation was confirmed by surgery in all patients.

\section{Conclusion}

Small bowel perforation is an acute emergency that requires a prompt and confident diagnosis and treatment. Surgery benefits of a complete and accurate radiological diagnosis and the radiologist has to know the direct and indirect signs of small bowel perforation on the different imaging technique to diagnose it and its origin.

\section{References}

1. Grassi R, Pinto A, Rossi G, et al: Conventional plain film radiology, ultrasonography and CT in jejuno-ileal perforations. Acta Radiol 39:52-56, 1998

2. Romano L, Pinto A: Imaging of Alimentary Tract Perforation. ${ }^{\circ}$ Springer International Publishing Switzerland, http://dx.doi.org/10.1007/978-3319-08192-2_6

3. Fang JF, Chen RJ, Lin BC, et al: Small bowel perforation: Is urgent surgery necessary? J Trauma 47:515-520, 1999

4. Frick Jr EJ, Pasquale MD, Cipolle MD: Small-bowel and mesentery injuries in blunt trauma. J Trauma 46:920-926, 1999

5. Scaglione M, Romano L, Bocchini G, et al: Multidetector computed tomography of pancreatic, small bowel, and mesenteric traumas. Semin Roentgenol 47:362-370, 2012

6. Iaselli F, Mazzei MA, Firetto C, et al: Bowel and mesenteric injuries from blunt abdominal trauma: A review. Radiol Med 120(1):21-32, 2015

7. Brody JM, Leighton DB, Murphy BL, et al: CT of blunt trauma bowel and mesenteric injury: Typical findings and pitfalls in diagnosis. Radiographics 20:1525-1537, 2000

8. Grassi R, Pinto A, Rossi G: Isolated pneumoretroperitoneum secondary to acute bowel infarction. Clin Radiol 55:321-323, 2000

9. Kimchi NA, Broide E, Shapiro M, et al: Non-traumatic perforation of the small intestine. Report of 13 cases and review of the literature. Hepatogastroenterology 49:1017-1022, 2002

10. Mazzei MA, Volterrani L: Nonocclusive mesenteric ischaemia: Think about it. Radiol Med 120(1):85-95, 2015

11. Pinto A, Reginelli A, Pinto F, et al: Radiological and practical aspects of body packing. Br J Radiol 87:20130500, 2014

12. Pinto A, Muzj C, Stavolo C, et al: Pictorial essay: Foreign body of the gastrointestinal tract in emergency radiology. Radiol Med 107:145-152, 2004

13. Allen GS, Moore FA, Cox Jr CS, et al: Hollow visceral injury and blunt trauma. J Trauma 45:69-78, 1998

14. Tirkes I, Sandrasegaran K, Patel AA, et al: Peritoneal and retroperitoneal anatomy and its relevance for cross-sectional imaging. Radiographics 32 (2):437-451, 2012

15. Gould RJ, Thorwarth WT: Retroperitoneal rupture of the duodenum due to blunt, non-penetrating abdominal trauma. Radiology 80:743-747, 1963

16. Grassi R, Romano S, Pinto A, et al: Gastro-duodenal perforations: Conventional plain film, US and CT findings in 166 consecutive patients. Eur J Radiol 50(1):30-36, 2004

17. Pinto A, Scaglione M, Pinto F, et al: Helical computed tomography diagnosis of gastrointestinal perforation in the elderly patient. Emerg Radiol 7:259-262, 2000

18. Coppolino F, Gatta G, Di Grezia G, et al: Gastrointestinal perforation: Ultrasonographic diagnosis. Crit Ultrasound J 5(suppl 1):S4, 2013

19. Chen H, Yang CC, Yeh YH: Role of upright chest radiography and ultrasonography in demonstrating free air of perforated peptic ulcers. Hepatogastroenterology 48(40):1082-1084, 2001

20. Kuzmich S, Burke CJ, Harvey CJ, et al: Sonography of small bowel perforation. Am J Roentgenol 201:W283-W291, 2013

21. Kim HC, Yang DM, Kim SW, et al: Gastrointestinal tract perforation: Evaluation of MDCT according to perforation site and elapsed time. Eur Radiol 24:1386-1393, 2014

22. Grassi R, Di Mizio R, Pinto A, et al: Comparative adequacy of conventional radiography, ultrasonography and computed tomography in sixty-one 
consecutive patients with gastrointestinal perforations. Radiol Med 91:747-755, 1996

23. Galia M, Agnello F, La Grutta L, et al: Computed tomography of bowel obstruction: Tricks of the trade. Expert Rev Gastroenterol Hepatol 19:1-11, 2015

24. Lo Re G, Cappello M, Tudisca C, et al: enterography as a powerful tool for the evaluation of inflammatory activity in Crohn's disease: Relationship of CT findings with CDAI and acute-phase reactants. Radiol Med 119(9): 658-666, 2014

25. Agrusa A, Romano G, Lo Re, G, et al: Hemoperitoneum following mild blunt abdominal trauma: First presentation of Crohn's disease. Acta Med Mediterr 30(2):315-318, 2014

26. Pinto A, Scaglione M, Giovine S, et al: Quaranta pazienti con perforazione gastrointestinale: Confronto tra la sede dei reperti TC spirale multidetettore di perforazione e la sede di perforazione riscontrata all'intervento chirurgico. Radiol Med 108:208-217, 2004

27. Lacalamita MC, Moschetta M, Mancini ME, et al: Role of CT in the diagnosis of jejunal-ileal perforations. Radiol Med 119(9):651-657, 2014

28. Levine MS, Scheiner JD, Rubesin SE, et al: Diagnosis of pneumoperitoneum on supine abdominal radiographs. Am J Roentgenol $156: 731-735,1991$

29. Keyting WS, McCarver RR, Kovarik JL, et al: Pneumatosis intestinalis: Mere concept. Radiology 76:733-741, 1961

30. Lassandro F, Valente $T$, Rea G, et al: Imaging assessment and clinical significance of pneumatosis in adult patients. Radiol Med 120(1):96-104, 2015
31. Ho MP, Cheung WK, Tsai KC, et al: Chilaiditi syndrome mimicking subdiaphragmatic free air in an elderly adult. J Am Geriatr Soc 62:2019-2021, 2014

32. Sessa B, Trinci M, Ianniello S, et al: Blunt abdominal trauma: Role of contrast-enhanced ultrasound (CEUS) in the detection and staging of abdominal traumatic lesions compared to US and CE-MDCT. Radiol Med 120(2):180-189, 2015

33. Pinto F, Valentino M, Romanini L, et al: The role of CEUS in the assessment of haemodynamically stable patients with blunt abdominal trauma. Radiol Med 120:3-11, 2015

34. Karahan OI, Kurt A, Yikilmaz A, et al: New method for the detection of intraperitoneal free air by sonography: Scissors maneuver. J Clin Ultrasound 32:381-385, 2004

35. Schueller G, Scaglione M, Linsenmaier $U$, et al: The key role of the radiologist in the management of polytrauma patients: Indications for MDCT imaging in emergency radiology. Radiol Med 120:641-654, 2015

36. Federle MP, Peitzman A, Krugh J: Use of oral contrast material in abdominal trauma CT scans: Is it dangerous? J Trauma 38(1):51-53, 1995

37. Lafferty B: Oral contrast omission in the computed tomographic evaluation of blunt abdominal trauma: A literature review. J Trauma Nurs 19(1): El-E4, 2012

38. Miki T, Ogata S, Uto M, et al: Multidetector-row CT findings of colonic perforation: Direct visualization of ruptured colonic wall. Abdom Imaging 29:658-662, 2004

39. Mindelzun RE, Jeffrey Jr RB, Lane MJ, et al: The misty mesentery on CT: Differential diagnosis. Am J Roentgenol 167:61-65, 1996 